Document downloaded from:

http://hdl.handle.net/10251/55102

This paper must be cited as:

Ricchiuti, AL.; Suárez Alvarez, I.; Barrera Vilar, D.; Rodríguez Cantó, PJ.; Fernandez-Pousa, CR.; Abargues, R.; Sales Maicas, S.... (2014). Colloidal Quantum Dots-PMMA Waveguides as Integrable Microwave Photonic Phase Shifters. IEEE Photonics Technology Letters. 26(4):402-404. doi:10.1109/LPT.2013.2295253.

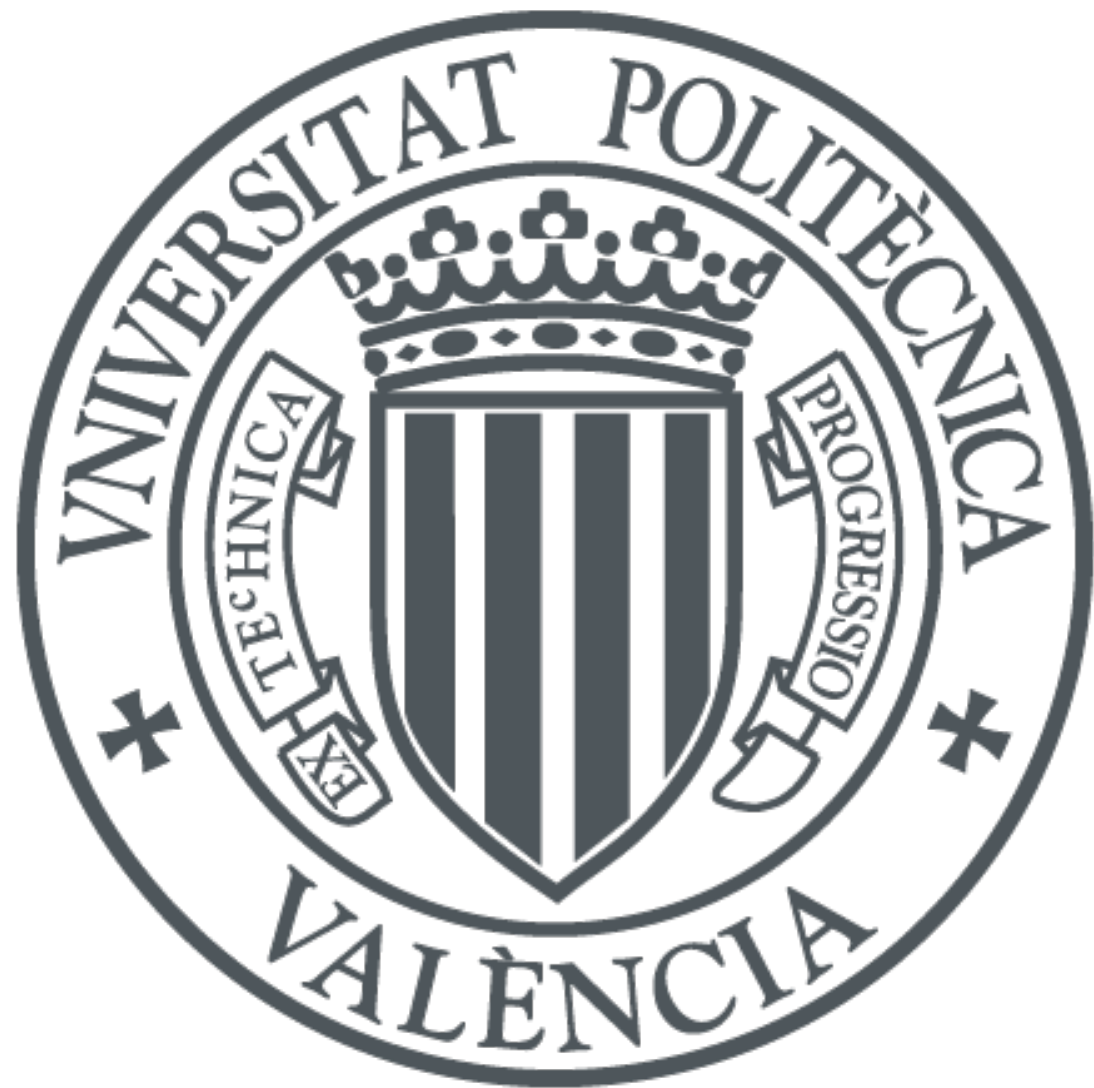

The final publication is available at

http://dx.doi.org/10.1109/LPT.2013.2295253

Copyright Institute of Electrical and Electronics Engineers (IEEE)

Additional Information

"(C) (C) 20xx IEEE. Personal use of this material is permitted. Permission from IEEE must be obtained for all other uses, in any current or future media, including reprinting/republishing this material for advertising or promotional purposes, creating new collective works, for resale or redistribution to servers or lists, or reuse of any copyrighted component of this work in other works." 


\title{
Colloidal Quantum Dots-PMMA Waveguides as Integrable Microwave Photonic Phase Shifters
}

\author{
A.L. Ricchiuti, I. Suárez, D. Barrera, P.J. Rodríguez-Cantó, C.R. Fernández-Pousa, R. Abargues, S. \\ Sales, J. Martínez-Pastor and J. Capmany
}

\begin{abstract}
A novel scheme for the control of microwave signals carried at optical wavelengths by use of $\mathrm{PbS}$ colloidal quantum dots (QDs) embedded in PMMA waveguides is presented. When these structures are pumped at wavelengths where $\mathrm{PbS}$ has efficient absorption (980 or $1310 \mathrm{~nm})$ a phase shift in a signal carried at $1550 \mathrm{~nm}$ is induced. Optimal conditions have been analyzed by studying the influence of the microwave signal and the waveguide structure. In a proof-of-concept experiment, a continuous phase shift up to $35^{\circ}$ at $25 \mathrm{GHz}$ has been demonstrated, with good thermal stability $\left(<2^{\circ}\right.$ at $\left.25 \mathrm{GHz}\right)$ when the samples are heated $20^{\circ} \mathrm{C}$ above room temperature. The potential benefits of the use of this active-waveguide technology in microwave photonics are due to the continuous scan of the phase delay, its high tuning speed, and its small size, which leads to the possibility of integration.
\end{abstract}

Index Terms-PMMA, PbS, integrable microwave phase shifter, planar waveguide, optical signal processing, colloidal QD.

\section{INTRODUCTION}

$\mathrm{M}$ ICROWAVE PHOTONICS (MWP) is a promising discipline relying on the interaction between microwaves or millimeter waves with optical wave signals. This emerging field has attracted great interest in the last decades not only from both the research community but also from the commercial sector, leading to new possibilities in a variety of application fields such as radar, communications, sensor systems or instrumentation [1], [2]. The reason of this success stems from the fact that the optical domain allows for the realization of key functionalities in microwave and millimeter wave systems which are either complex or even not directly possible in the radio-frequency (RF) domain, such as high purity microwave signal and waveform generation,

Manuscript received November 5, 2013; revised December 5, 2013; accepted December 10, 2013. Date of publication xxx; date of current version December 5, 2013. The authors wish to acknowledge the financial support of the Infraestructura FEDER UPVOV08-3E-008, FEDER UPVOV10-3E-492, the financial support given by the Research Excellency Award Program GVA PROMETEO 2013/012, "Next generation Microwave Photonic technologies," and the Spanish MCINN through the projects TEC2011-29120-C05-01,-02, and -05.

A.L. Ricchiuti, D. Barrera, S. Sales, and J. Capmany are with iTEAM Research Institute, Optical Quantum and Communications Group, Universidad Politécnica de Valencia, 46022 Valencia, Spain (amric1@iteam.upv.es).

I. Suárez, P.J. Rodríguez-Cantó, R. Abargues, and J. Martínez-Pastor are with UMDO, Instituto de Ciencia de los Materiales, Universidad de Valencia, 46071 Valencia, Spain (isaac.suarez@uv.es).

C.R. Fernández-Pousa is with Departamento de Ingeniería de Comunicaciones, Universidad Miguel Hernández, 03202 Elche, Alicante, Spain (c.pousa@umh.es). tunable and reconfigurable adaptive filtering, optical beam forming, optoelectronic oscillation, analog to digital conversion and radio over fiber communication systems [3].

A key component in most of these applications is the microwave photonics phase shifter, for which several schemes have been reported in the literature. In [4], for instance, a phase shifter based on wavelength conversion in a distributed feedback laser is demonstrated, and in [5] the phase tuning was achieved by changing the optical carrier wavelength by means of stimulated Brillouin scattering (SBS) in optical fiber. For narrowband phase shift, silicon-on-insulator (SOI) ring resonators have been used as widely tunable RF phase shifters [6]. Furthermore, phase shifts can also be achieved based on slow- and fast-light effects induced by coherent population oscillations (CPOs) in semiconductor optical amplifiers (SOAs) [7]. However, and although these technologies permit an efficient control of the microwave phase, they also show some drawbacks that may limit their practical applications. For example, the SBS-based phase shifter requires both long fiber lengths and high pump powers; the use of CPOs in SOAs adds both noise and distortion; and the bandwidth of the SOI ring resonator is restricted to a few $\mathrm{GHz}$.

In this paper we propose a novel technology enabling integrable microwave photonic phase shifters that can overcome the previous limitations. The proposed technology is based on nanocomposites fabricated by the dispersion of colloidal quantum dots (QDs) in polymethylmethacrylate (PMMA) matrix. A phase shift is induced over microwave signals carried at $1550 \mathrm{~nm}$ when the waveguide is pumped at wavelengths where the nanoparticles show significant absorption (1310 or $980 \mathrm{~nm}$ ). Moreover, the measurements show good thermal stability even if the samples are heated 20 ${ }^{\circ} \mathrm{C}$ above room temperature. These new structures show potential benefits such as high tuning speed, continuous scan of the phase delay, small size, capability of integration and low tuning losses.

\section{WAVEGUIDE FABRICATION AND MEASUREMENT SETUP}

The samples used in this work are based on nanocomposites fabricated by the dispersion of PbS QDs in a poly(methyl methacrylate) (PMMA) matrix. This multicomponent material has a great interest nowadays because it joins the properties of the nanostructures (QD) [8] with the technological feasibility of the matrix (PMMA) [9] and hence it can act as the core of a planar waveguide whose active properties can be tuned by 
controlling the size, concentration and type of the embedded QDs [10]. In particular, saturation of the absorption of pumped QDs [11] is expected to vary the group index of signals modulated at $1550 \mathrm{~nm}$ through $\mathrm{CPO}$, so that the PbS-PMMA waveguide acts as a tunable microwave photonic phase shifter. After photo-detection, the group index variation is translated into a phase change $(\Delta \varphi)$ in the microwave signal that can be expressed as:

$$
\Delta \varphi=\Delta k L=\frac{\Omega}{c} \Delta n_{g} L
$$

being $k$ the wave vector of the signal, $c$ the speed of light in vacuum, $\Omega$ the angular frequency of the RF tone, $n_{g}$ and $L$ the group index and the length of the medium, respectively.

The PbS QDs used in this work were synthesized following the procedure developed by Peng's group as explained in [10]. Since the objective is to demonstrate phase shift by the absorption of these nanocrystals at telecommunication windows, the size of the QD was tuned to show efficient absorption in these wavelengths [10]. The inset in Fig. 1 shows the absorption spectra of the PbS QDs and the structure of the waveguide sample, where the exciton peak is located around $1300 \mathrm{~nm}$. For shorter wavelengths, absorption progressively increases and for longer ones it progressively decreases being virtually zero beyond $1600 \mathrm{~nm}$.

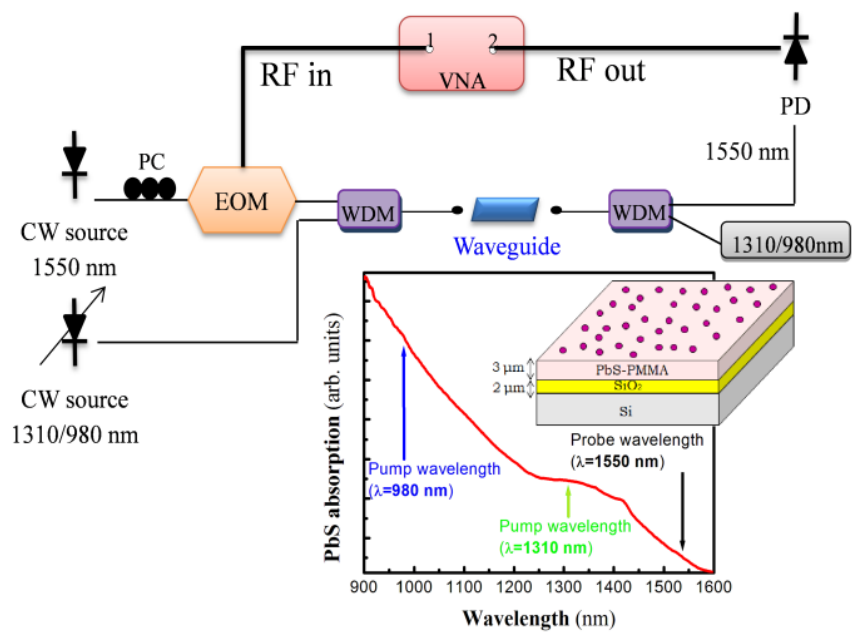

Fig. 1. Layout of the measurement setup. The inset shows the structure of the sample and the absorption spectrum of the colloidal PbS QDs.

The nanocomposite was fabricated by mixing the QD colloidal solution with PMMA. Both the polymer and QDs were first dissolved in toluene to reach a good dispersion of the nanoparticles in the matrix [10] with filling factors ( $f f)$, of $\mathrm{PbS}$ in the PMMA ranging from $10^{-3}$ and $10^{-2}$ in order to obtain a compromise between QD excitation and waveguiding [10]. Here $f f$ is defined as the volume ratio between QDs and nanocomposite. Finally, the waveguides were completed by depositing $3 \mu \mathrm{m}$ of nanocomposite on a $\mathrm{SiO}_{2} / \mathrm{Si}$ substrate $(2$ $\mu \mathrm{m} \mathrm{SiO}_{2}$ ).

Figure 1 shows our characterization setup. The intensity of a continuous-wave $(\mathrm{CW})$ laser at $1550 \mathrm{~nm}$ was electro-optically modulated (EOM) with a microwave tone $(10 \mathrm{MHz}-25 \mathrm{GHz})$ generated by a vector network analyzer (VNA). At the output of the EOM, the double-side band signal was mixed with a $\mathrm{CW}$ pump at 1310 or $980 \mathrm{~nm}$ by means of a wavelength division multiplexer (WDM). Then, the signal was injected at the input edge of the planar waveguide by use of a lensed fiber, and the output light was collected with another lensed fiber, separating the $1550 \mathrm{~nm}$ modulated signal from the pump signal by means of a second WDM. The optical 1550-nm signal was photo-detected (PD) and, finally, the amplitude and phase waveguide response was analyzed by monitoring the scattering parameter $S_{21}$ which relates the RF detected signal with the microwave modulating signal. The phase shift in microwave signals modulated at $1550 \mathrm{~nm}$ is created by varying the power and the wavelength of the $\mathrm{CW}$ pump [11].

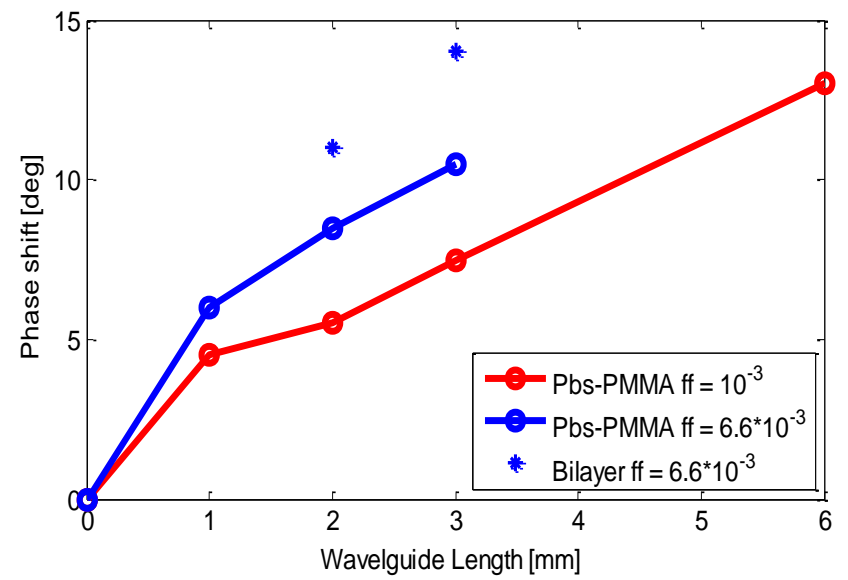

Fig. 2. Phase shifts at $25 \mathrm{GHz}$ as function of waveguide length for 1310-nm pumped, PbS-PMMA waveguides with $f f=10^{-3}$ (red curve) and $f f=6.6 \cdot 10^{-3}$ (blue curve) and for bilayer samples with $f f=6.6 \cdot 10^{-3}$ (blue dots).

\section{EXPERIMENTAL RESULTS}

Initially, the waveguide has been pumped with a $1310 \mathrm{~nm}$ laser source and a phase shift has been induced in the $1550 \mathrm{~nm}$ carrier probe. According to (1) the phase shift is proportional to the microwave frequency $\Omega$, the waveguide length and the induced group index variation. The latter can be controlled by the pump wavelength, the concentration of QDs (or ff) and the length of the structure [11]. Figure 2 shows the phase shifts measured at $25 \mathrm{GHz}$ at an estimated pump power coupled to the waveguide of $0.085 \mathrm{~mW}$ in samples with different concentrations of $\mathrm{PbS}\left(f f=10^{-3}, 6.6 \cdot 10^{-3}\right)$ and waveguide lengths $(L=1,2,3,6 \mathrm{~mm})$, confirming that the phase shift in the microwave signal increases with $f f$ and the waveguide length. The deviation of the linear proportionality between phase shift and waveguide length predicted by (1) is attributed to the high absorption of the PMMA matrix, which results in pump depletion. Besides, for high $f f$, the waveguide length is limited because at high concentrations the absorption at the probe wavelength also becomes significant (see Fig. 1). Indeed, for a sample with $f f=6.6 \cdot 10^{-3}$ and $L=6 \mathrm{~mm}$ (blue line), we found that the signal to noise ratio at the input of the VNA was not sufficiently high to perform the measurement.

Nevertheless, it is possible to alleviate this limitation by using a structure consisted of a PMMA cladding deposited on top of the QD nanocomposite. This bilayer structure, first 
proposed in [12], improves the $1.55-\mu \mathrm{m}$ probe confinement and thus provides an increase of the received power. We have checked that the microwave signal is improved almost $20 \mathrm{~dB}$ with a $2 \mathrm{~mm}$ length waveguide if a PMMA cladding with a thickness of $3 \mu \mathrm{m}$ is deposited on top of the nanocomposite ( $f f$ $\left.=6.6 \cdot 10^{-3}\right)$. Moreover, by comparing the phase shift obtained with the bilayer (blue dots of Fig. 2) and the monolayer (blue circles of Fig. 2) waveguides it can be concluded that the PMMA cladding layer improves the figure of merit.

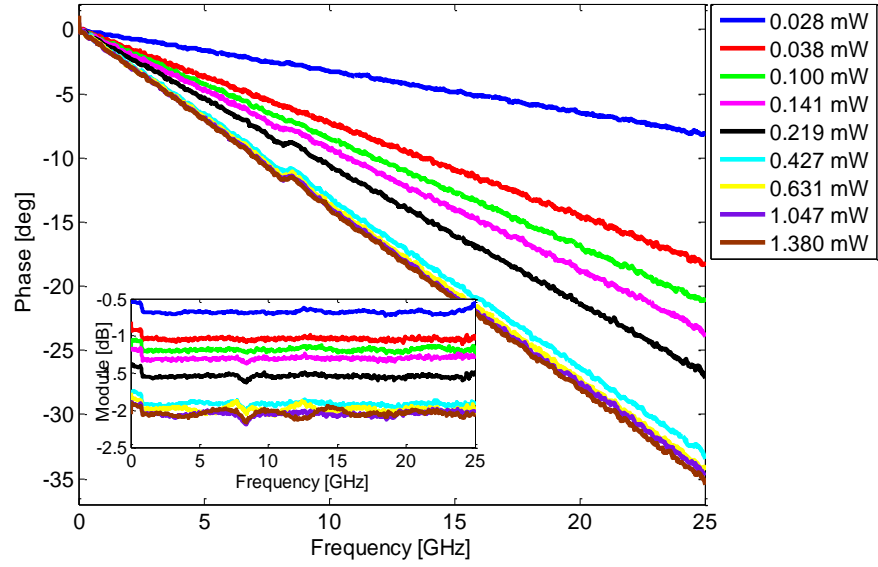

Fig. 3. Phase shifts in a $4 \mathrm{~mm}$ long bilayer PbS-PMMA waveguide $\left(f f=10^{-2}\right)$ for different pump powers. The inset shows the variations in RF amplitude.

In order to show that by pumping the waveguides at shorter wavelengths the phase shift in the microwave signal could be improved, the experiment was repeated by pumping the proposed bilayer samples at $980 \mathrm{~nm}$. The results are shown in Fig. 3. The experiment was performed in a bilayer waveguide with $4 \mathrm{~mm}$ length and $f f=10^{-2}$. The higher the pump power the larger the phase-shift, saturating to values up to $35^{\circ}$ at 25 $\mathrm{GHz}$, due probably to nanocrystal saturation [11]. Also, it is important to note that the variations in $\mathrm{RF}$ amplitude are less than $2 \mathrm{~dB}$, as illustrated in the inset of Fig. 3.

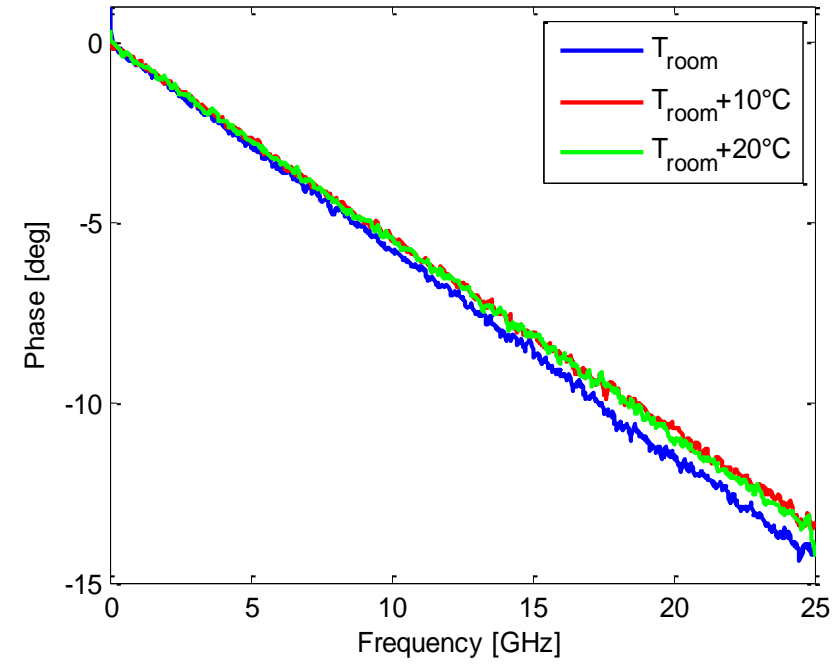

Fig. 4. Phase shifts in a $2 \mathrm{~mm}$ long bilayer waveguide with $\mathrm{ff}=6.6 \cdot 10^{-3}$ for different temperatures.

Finally, the thermal stability of these active waveguides was studied by increasing the temperature of the sample $\sim 10{ }^{\circ} \mathrm{C}$ and $\sim 20^{\circ} \mathrm{C}$ over room temperature by using a ceramic resistor. Figure 4 shows the results in a $2 \mathrm{~mm}$ long bilayer PbS-PMMA waveguide with $f f=6.6 \cdot 10^{-3}$, while the inset illustrates the variations in RF amplitude.

This characterization of the device shows that negligible changes in the phase shift are provoked by heating the samples $\left(<2^{\circ}\right.$ at $\left.25 \mathrm{GHz}\right)$. Besides, the variations of the RF amplitude are being measured given a value of less than $0.25 \mathrm{~dB}$.

\section{CONCLUSIONS}

In this paper, an integrable microwave photonic phase shifter based on active PMMA planar waveguides has been proposed and experimentally demonstrated. The polymer is doped with $\mathrm{PbS}$ QDs with the intention to provide a saturation absorption effect at infrared wavelengths. Under these conditions, the group speed refractive index is modified, and therefore the PbS-PMMA waveguide acts as a tunable microwave photonic phase shifter. The amount of phase shift can be fine tuned through the waveguide length, the pump wavelength or the QDs concentration. Besides, we have proposed a bilayer structure consisted of a PMMA cladding deposited on the QD nanocomposite with the intention to reduce the propagation losses. In a series of proof-of-concept experiments, phase shifts up to $35^{\circ}$ at $25 \mathrm{GHz}$ have been obtained for bilayer waveguides pumped at $980 \mathrm{~nm}$. It was also shown that these devices show a good thermal stability.

In summary, we have presented a novel technology based on hybrid organic-inorganic materials for its use as integrated MWP phase shifters. The potential benefits of the proposed devices are the continuous scan of the phase delay, the low tuning losses its thermal drift resilience, its high tuning speed, and its small size, which leads to the possibility of integration.

\section{REFERENCES}

[1] J. Capmany and D. Novak, "Microwave photonics combines two worlds," Nature Photon., vol. 1, pp. 319-330, Jun. 2007.

[2] A. Seeds, "Microwave photonics," IEEE Trans. Microw. Theory Techn., vol. 50, no. 3, pp. 877-887, Mar. 2002.

[3] J. Capmany et al., "Microwave Photonic Signal Processing," J. Lightw. Technol, vol.. 31, no. 4, pp. 571-586, Feb. 2013.

[4] M. Fisher and S. Chuang, "A microwave photonic phase-shifter based on wavelength conversion in a DFB laser," IEEE Photon. Technol. Lett,. vol. 18 , no. 16, pp. 1714-1716, Aug. 2006.

[5] A. Loayssa, F. J. Lahoz, "Broad-band RF photonic phase shifter based on stimulated Brillouin scattering and single-sideband modulation," IEEE Photon. Technol. Lett., vol. 18, no. 1, pp. 208-210, Jan. 2006.

[6] Q. Chang et al., "A tunable broadband photonic RF phase shifter based on a silicon microring resonator," IEEE Photon. Technol. Lett., vol. 21, no. 1, pp. 60-62, Jan. 2009.

[7] S. Sales et al., "Slow and fast light effects and their applications to Microwave Photonics using semiconductor optical amplifiers" IEEE Trans. Microw. Theory Tech./ J. Lightw. Technol., Joint Special issue on Microwave Photonics, vol. 58, pp. 3022-3038, 2010.

[8] V.I. Klimov, "Nanocrystal quantum dots: from fundamental photophysics to multicolor lasing," Los Alamos Science, vol. 28, no. 60, pp. 214-220, Sep. 2003.

[9] M.A. Uddin and H.P. Chan, "Materials and process optimization in the reliable fabrication of polymer photonic devices," J. Optoelectron. Adv. M., vol. 10, pp. 1-17, Jan. 2008.

[10] I. Suárez et al., "Photoluminescence waveguiding in CdSe and CdTe QDs-PMMA nanocomposite films," Nanotechnology, vol. 22. pp. 435202, Sep. 2011.

[11] V. I Kilmov et al., "Single-exciton optical gain in semiconductor nanocrystals," Nature. vol. 447, pp. 441-446, May 2007.

[12] H. Gordillo et al., "Quantum-dot double layer polymer waveguides by evanescent light coupling," J. Lightwave Technol,. vol. 31, no. 15, pp. 2515-2525, Aug. 2012. 